\title{
HYBRID CLASSIFIER FOR BRAIN ABNORMALITY DETECTION IN BRAIN MRI
}

\author{
Associate Professor, Electronics and Communication Engineering, Kalasalingam \\ Academy of Research and Education, Tamilnadu, (India). \\ E-mail: lakshmi@klu.ac.in \\ ORCID: http://orcid.org/0000-0002-6744-7048
}

Thangadurai Arivoli

Professor/Principal, Electronics and Communication Engineering,

Vicram College of Engineering, Tamilnadu, (India).

E-mail: t.arivoli@gmail.com

ORCID: http://orcid.org/0000-0003-1612-1029

M. Pallikonda Rajasekaran

Professor/ Director of Controller of Examination,Electronics and communication EngineeringKalasalingam Academy Of Research and Education, Tamilnadu, (India).

E-mail:m.p.raja80@gmail.com

ORCID: https://orcid.org/0000-0001-6942-4512

\begin{abstract}
Assistant professor, Electronics and Communication Engineering, Kalasalingam Academy of Research and Education, Krishnankoil, Tamilnadu, (India).

E-mail: bhuvaneswary.n@klu.ac.in ORCID: http://orcid.org/0000-0001-6400-6602
\end{abstract}

S. Sathya

PG student,Eectronics and Communication Engineering, Kalasalingam Academy Of

Research and Education, Krishnanloil, Tamilnadu, (India).

E-mail: ssathya08nov@gmail.com

ORCID: https://orcid.org/0000-0002-1440-663X

Recepción: 25/10/2019 Aceptación: 20/10/2020 Publicación: 30/11/2021

\section{Gitación sugerida:}

Lakshmi, A., Arivoli, T., Rajasekaran, M., Bhuvaneshwary, N., y Sathya, S. (2021). Hybrid classifier for brain abnormality detection in brain MRI. 3C Tecnología. Glosas de innovación aplicadas a la pyme, Edición Especial, (noviembre, 2021), 125-153. https://doi.org/10.17993/3ctecno.2021.specialissue8.125-153 


\section{ABSTRACT}

Brain abnormality detection is very essential now a days. Brain abnormality includes both massive abnormal growth of cells called tumour and blood lump in a veins or artery. If The abnormalities are detected at the earlier stages it would help to save life. The symptoms for the abnormalities are same and vary with patient aging and severity of the abnormality. The algorithm which works for tumor can not be used for the detection of abnormality caused in blood lumps of veins and artery. Normally the tumor is the massive growth of the cell and the features can be extracted and applied for the classifier to identify the severity of the abnormality. But the detection of abnormality caused in vein or artery due to blood lumps are very difficult to identify and feature extraction is also difficult. A sophisticated algorithm should be used for identifying the blood lumps. This paper deals with hybrid classifier (SVM and ANFIS) for detecting the abnormalities such as tumour as well as stroke. Till now separate algorithms are used for detecting tumour or stroke from brain MR image. In our proposed work it is possible to identify stroke or tumour with same algorithm by using different hybrid classifiers. The proposed system helps the physicians to diagnose human brain stroke. Accuracy of 0.999, sensitivity 0.38, specificity 0.86, PPV 0.91, NPV 0.99 is obtained by ANFIS classifier. Three quantitative events to calculate brain tumor of average segmentation results: Similarity Index (SI), Overlap fraction (OF), and Extra Fraction $(\mathrm{EF})$ is $0.776194,0.775198,0.222213$ is obtained by SVM classifier.

\section{KEYWORDS}

SVM, ANFIS, Brain Stroke, Tumour. 


\section{INTRODUCTION}

\section{Brain Stroke}

Stroke otherwise called as "Brain Attack" that happens in the brain rather than the heart. A stroke or "brain assault" occurs:

- Due to blood lump in a veins or artery.

- Due to blood vein breakage, interfering with blood stream to a region of the brain. As it happens, brain cells start to expire. When brain cells die during a stroke, functions such as speech, movement and memory guarded by that part of the brain are vanished. The lost of function depends on the location of the stroke and on its severity i.e., the amount of brain cell loss. Several persons get well entirely from fewer severe strokes, while other strokes can be considered life threatening. Opposite treatments are required for both strokes namely ischemic stroke and hemorrhagic stroke.
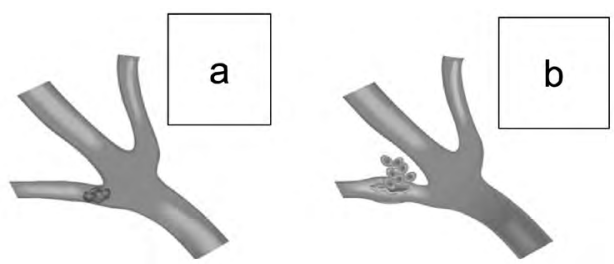

Figure 1. Brain Attack. a) Ischemic, b) Hemorrhagic Stroke.

Suorce: own elaboration.

\section{$\underline{\text { Brain Tumour }}$}

A tumour is unusual tissue that develops by unrestrained cell splitting up. Healing methods vary depending on the following factors such as tumour type, size, location, age and medical health of the person. Curing method may be therapeutic or attention on discharging indications.

Figure 2. MRI Brain Tumours.

Suorce: own elaboration.

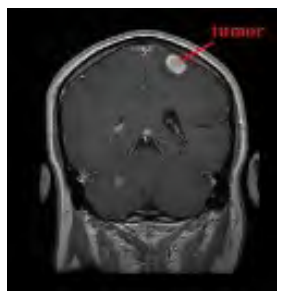


Primary brain tumours may be benign (non-cancerous) or malignant (cancerous). A benign brain tumour develops gradually and malignant brain tumour grows rapidly, has unequal boundaries, and spreads to close by brain areas. Sometimes they are called brain cancer. Whether a brain tumour is benign, malignant are potentially life-threatening. Grade means the way tumour cells look under the microscope and is a suggestion of aggressiveness. If the tumours are detected at near the beginning stage there is the opportunity of saving the life.

Cerebrovascular accident (CVA) is a damage caused because of functionality of brain due to lack of flow of blood (Adams et al., 2005). This smash up is due to either ischemic or hemorrhage. Blockage of blood in blood vessels inside the brain is known as Ischemic stroke. Ischemicstroke results in about $80 \%$ deaths caused by all strokes. Bleeding of blood in blood vessels inside the brain is called Hemorrhagic stroke (Udupa \& Samarasekera, 1996). In any case, the damaged area of the brain cannot function in a normal way. When brain cells expire, the tasks controlled by the region of the brain are vanished.

Kharat and Kulkarni (2012) presented the classification of brain tumors using neural networks. Due to complexity and variance, the MR image classification was a difficult task. Generally two neural network techniques were used.

The taken out features are categorized using Multi Layer Perceptron (MLP) (Amutha \& Rajagopalan, 2013). Feature lessening is expertise by grade of the features using Information gain. Multilayer Perceptron (MLP) is used to categorize the extracted features.

Mid line of brain helps in measuring the brain's amount of equilibrium. Here, the brain's amount of equilibrium was determined using MLS technique (Dzialowski et al., 2012). It can detect early identification of ischemic stroke to improvise effectiveness and correctness of medical practice.

Hema and Bhavani (2013) suggested SVM, K-NN, ANN and decision tree classification with accuracy of $98 \%, 97 \%, 96 \%$ and $92 \%$. This system helped the physicians for enhanced detect of human brain stroke, for advance action. The brain area affected by abrasion can be accurately detached from the brain image. It improves the exactness in recognition of ischemic stroke. 
An improved watershed image segmentation technique was existing by Bala (2012). Watershed Transformation based an edge detection algorithm was used for image segmentation and makes use of gradient operators in mathematical morphology. But the drawback is over segmentation. To avoid over segmentation, Prewitt's edge detection operator with noise removal techniques and image enhancement wereestablished and the trouble of over segmentation was also reduced.

Mahmoud-Ghoneim et al. (2003) developed in intensity-based analysis. Features of different types can be calculated for texture analysis, such as co-occurrence matrix.

Udupa and Samarasekera (1996) presented the purpose fuzzy connectedness procedure has been applied. The grades of work have 0.750, 0.706, and 0.107 SI, OF, and EF respectively. Our proposed work has higher performance than the work by Udupa and Samarasekera (1996).

From the literature survey it was understood that fully automated system to detect stroke or tumour will assist the doctor very much. Till now separate algorithms are used for detecting tumour or stroke from brain MR image. In our proposed work it is possible to identify stroke or tumour with same algorithm by using different hybrid classifiers.

\section{MATERIALS AND METHODS}

The main objective is to detect the presence of Brain Abnormalities in MRI by Hybrid (SVM and ANFIS) classifiers. The Brain Abnormalities such as stroke and tumor images are classified by Image Intensity Threshold Level. After the classification, images are preprocessed. After preprocessing, the features are extracted and the Hybrid classifiers are used to segmentation and detection of Brain Abnormalities and also performance analysis if performed. 


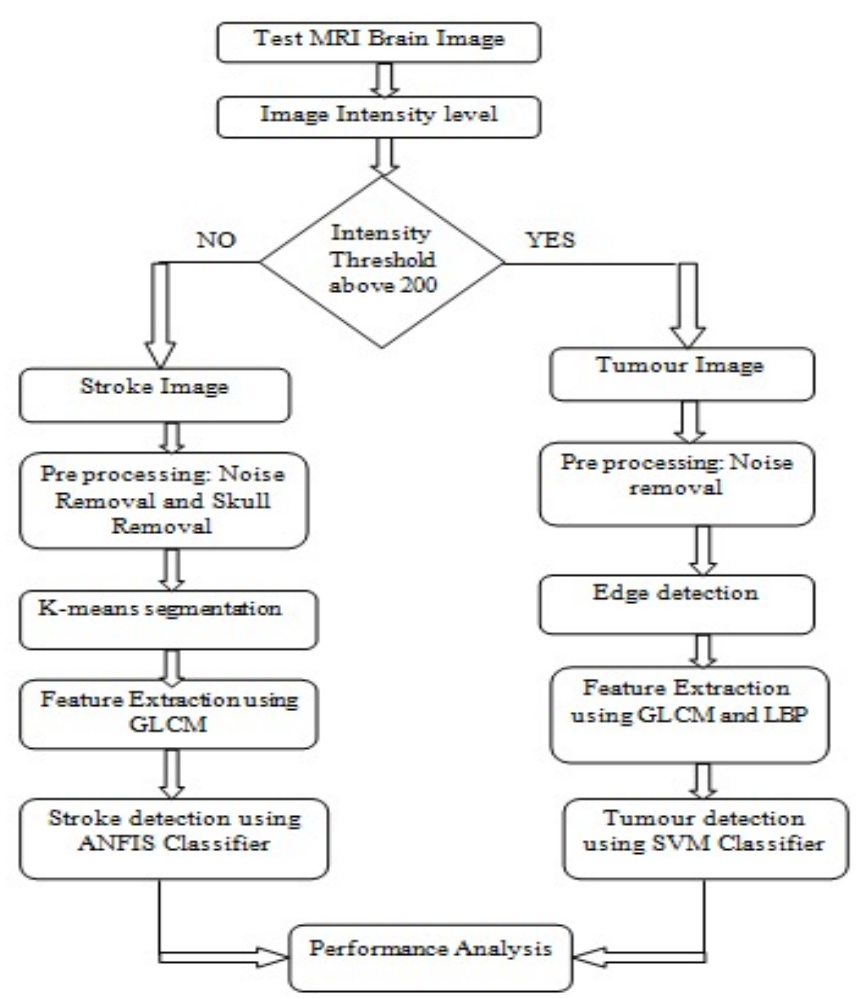

Figure 3. Proposed Data flow diagram.

Suorce: own elaboration.

\subsection{PROPOSED METHOD FOR IDENTIFYING TUMOUR}

If the tested MRI image intensity level is above 200, it will detect tumour otherwise to detect stroke as shown in the block diagram in Figure 4 the following steps will be carried in tumor segmentation:

- Pre-processing-Noise removal using Anisotropic filter.

- Canny Edge detection.

- Feature Extraction using GLCM matrix and LBP.

- Tumor detection using SVM classifier. 


\subsubsection{PRE-PROCESSING-NOISE REMOVAL USING ANISOTROPIC FILTER}

It is necessary to reduce image noise exclusive of removing noteworthy elements of the image content such as edges, lines or other elements those are significant for the understanding of the image.

The dataset of numerous diverse images of the brain tumour have studied and through testing threshold appropriate for those images are identified. Then, for same intensity ranges in all the images, the maximum and the smallest intensities are restricted to the range $[0,255]$. Then anisotropic diffusion is defined as,

$$
\frac{\partial I}{\partial t}=\operatorname{div}(c(x, y, t) \nabla I)=\nabla c \bullet \nabla I+c(x, y, t) \Delta I
$$

where $\Delta$ means the Laplacian, $\nabla$ signifies the gradient, div $(\ldots)$ is the divergence operator and $\mathrm{c}(\mathrm{x}, \mathrm{y}, \mathrm{t})$ is the diffusion coefficient. $\mathrm{G}(\mathrm{x}, \mathrm{y}, \mathrm{t})$ organizes the degree of diffusion and is regularly preferred as a the image gradient so as to conserve boundaries in the image.

Pietro Perona and Jitendra Malik lead the way the knowledge of anisotropic diffusion in 1990 and projected two functions for the diffusion coefficient:

$$
\begin{aligned}
& c(\|\nabla I\|)=e^{-(\|\nabla I\| / K)^{2}} \\
& c(\|\nabla I\|)=\frac{1}{1+\left(\frac{\|\nabla I\|}{K}\right)^{2}}
\end{aligned}
$$

Where $\mathrm{K}$ manages the sensitivity to boundaries. The noise removed brain MR image byanisotropic filtering is shown in Figure 4 . 


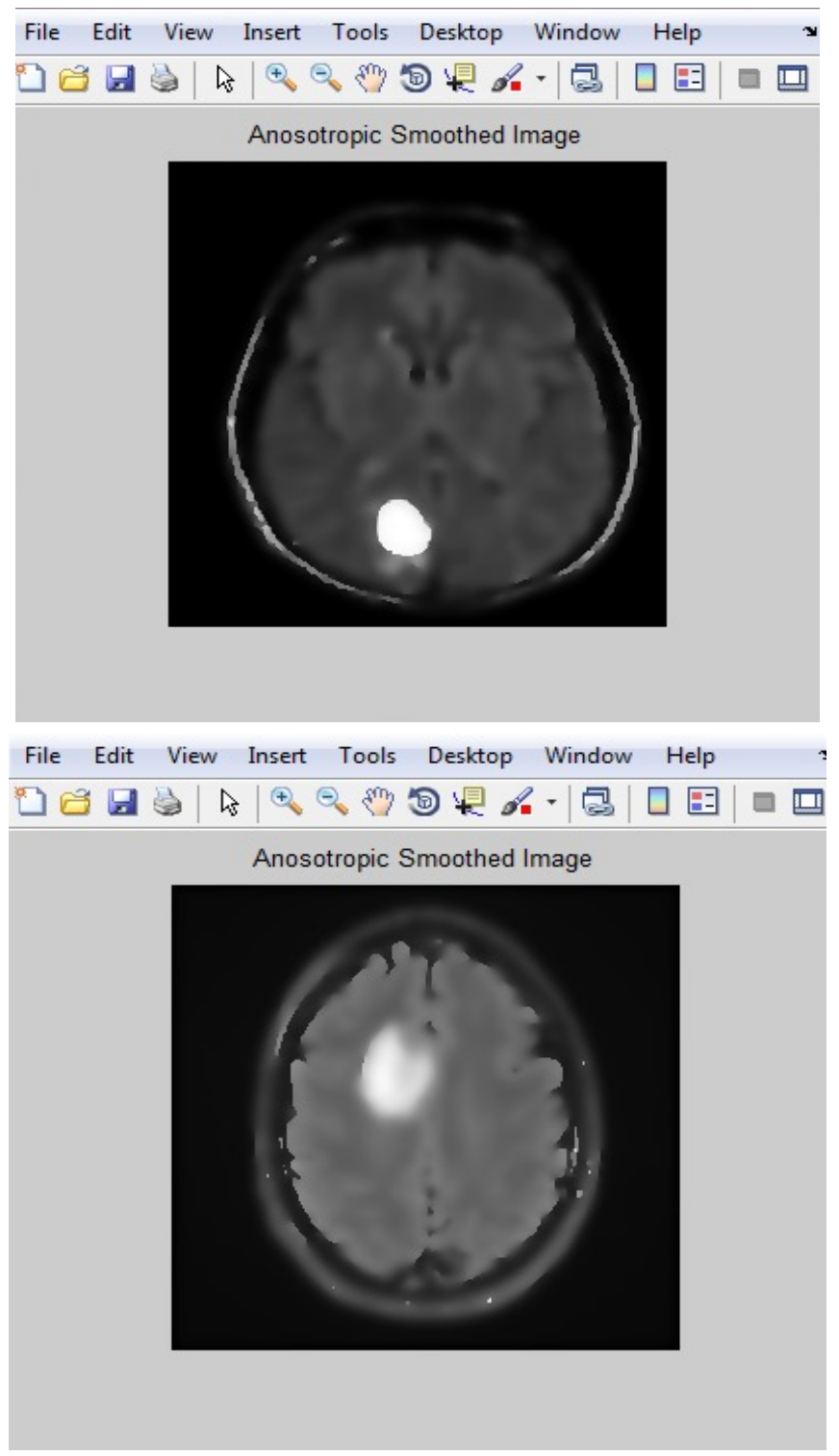

Figure 4. Noise Removed brain MR image using Anisotropic filter.

Suorce: own elaboration.

The noise filtered image, the intensity gradient of the image is found out. This is done by applying pair of convolution mask along the $\mathrm{x}$ and $\mathrm{y}$ directions. Then gradient strength and direction is found out using the formula.

$$
G=\sqrt{G_{x}{ }^{2}+G_{y}{ }^{2}} \quad \theta=\arctan \left(\frac{G_{y}}{G_{x}}\right)
$$


The direction was rounded off to any possible angle values namely (0, 45, 90 and 135). By doing this operation pixel which belongs to edges of theimage will be maintained and the pixel which are not belong to edges of the image are removed. Canny provides two thresholds namely upper and lower. If the pixel gradient is larger than upper threshold value, that pixel is established as edge. If the pixel gradient is lesser than the lower threshold value, then it is discarded. The Canny Edge detected brain MRI is shown in Figure 5.

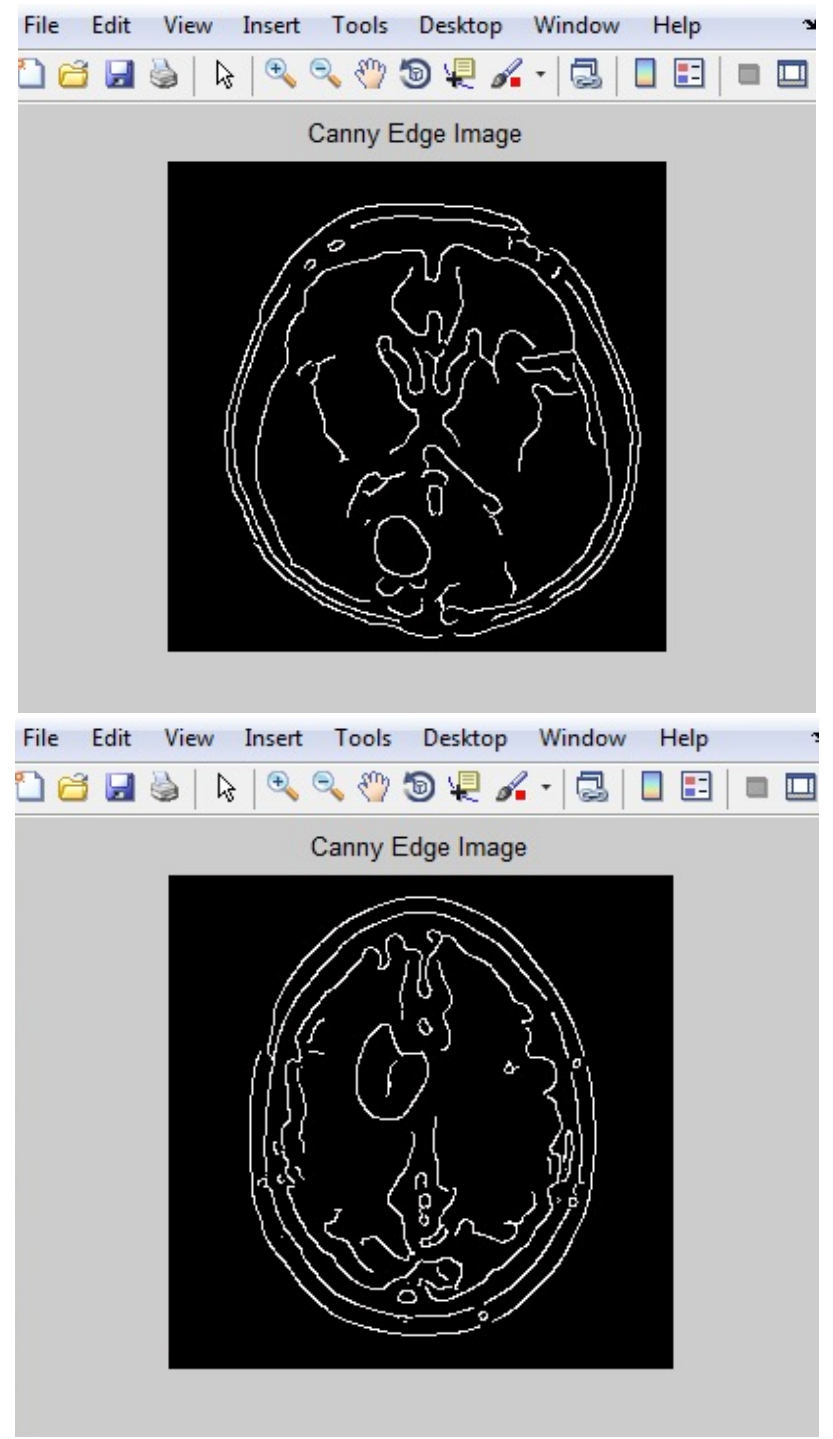

Figure 5. Canny Edge Detection.

Suorce: own elaboration. 


\subsubsection{FEATURE EXTRACTION USING GLCM MATRIX AND LBP}

The replicating pattern of local dissimilarity in image intensity is called consistency. This technique can be in consistency studies co-occurrence matrix. The information of pixel strength was expected from image that can be done using co-occurrence matrix. This pattern will compare by excellent consistency than by common consistency. The gray level co-occurrence matrix (GLCM) technique is a method of removing second order values stability elements. Though, the performance of a certain GLCM based on aspect, on top of the position the consistency characteristics; hinge on on the amount of gray levels applied. The subsequent representations are: mean value of $\mathrm{P}$ is represented as $\mu$. were the means and standard deviations of Px and Py are denoted as $\mu \mathrm{x}, \mu \mathrm{y}, \sigma \mathrm{x}$ and $\sigma \mathrm{y}$. The size of the occurrence matrix is represented as G. At this time the sum of rows and columns of co-occurrence matrix is identical. The subsequent GLCM characteristics are used in our study attempt: angular second moment, contrast, entropy, and correlation, sum of squares, variation entropy, inverse distinction moment, inertia, cluster importance, cluster shade, energy, homogeneity, distinction and diversity in variance. They are clear in following Equations.

1) Angular second moment (ASM):

$$
A S M=\sum_{i=0}^{G-1 G-1} \sum_{j-0}^{G-1}\{p(i, j)\}^{2}
$$

ASM is a determined similarity of the image. A standardized image will enclose simply some gray intensities, GLCM provides simply a rare except comparatively elevated ideals of $\mathrm{P}(\mathrm{i}, \mathrm{j})$.

2) Contrast:

$$
\text { Contrast }=\sum_{n-0}^{G-1} n^{2}\left\{\sum_{i-1}^{G} \sum_{j-1}^{G} p(i, j)\right\},|i-j|=n
$$

It is determined for the limited deviation in an image. 
3) Inverse distinction moment (IDM):

$$
I D M=\sum_{i=0}^{G-1 G-1} \sum_{j-0}^{G-(i-j)^{2}} p(i, j)
$$

IDM as well as inclined with the similarity of the image.

4) Entropy:

Entropy $=-\sum_{i=0}^{G-1 G-1} \sum_{j=0}^{G-1} p(i, j) \times \log p(i, j)$

It assesses the disarray or density of an image.

5) Correlation:

Correlation $=\sum_{i=0}^{G-1 G-1} \sum_{j-0}^{-1} \frac{\left\{(i \times j) \times p(i, j)+\left(\mu_{x} \times \mu_{y}\right)\right\}}{\sigma_{x} \times \sigma_{y}}$

It is calculated for gray level linear reliance

6) Sum of squares, variance:

$\operatorname{var}=\sum_{i-0}^{G-1 G-1} \sum_{j-0}^{G-1}(i-\mu)^{2} p(i, j)$

It is calculated for gray level dissimilarities at an assured distance, $\mathrm{d}$.

7) Variation entropy:

$$
D_{E n t}=-\sum_{i-0}^{G-1} p_{(x+y)}(i) \log \left(p_{x+y}(i)\right)
$$

Variation entropy is an evaluate, of histogram satisfied and rational rate among two images. 
8) Inertia:

$$
\text { Inertia }=\sum_{i=0}^{G-1 G-1} \sum_{j=0}^{G}(i-j)^{2} \times p(i, j)
$$

The inertia specifies the portion of gray scales in the image.

9) Cluster shade:

$$
\text { Shade }=\sum_{i=0}^{G-1 G-1} \sum_{j-0}^{G-1}\left\{i+j-\mu_{x}-\mu_{y}\right\}^{3} \times p(i, j)
$$

The image is not symmetric while darkness is elevated.

10) Cluster prominence:

$$
\operatorname{Pr} \text { om }=\sum_{i=0}^{G-1 G} \sum_{j-0}^{G-1}\left\{i+j-\mu_{x}-\mu_{y}\right\}^{4} \times p(i, j)
$$

Cluster Prominence specifies the image is not symmetric while prominences are elevated.

11) Energy:

$$
\text { Energy }=\sum_{i=0}^{G-1 G-1} \sum_{j-0}^{G-1} p(i, j)^{2}
$$

The energy of a surface explains the evenness of the surface. Energy is one for a stable image.

12) Homogeneity:

$$
\text { Homogenity }=\sum_{i=0}^{G-1 G-1} \sum_{j-0} \frac{p(i, j)}{1+|i-j|}
$$

Homogeneity proceeds a worth that events the nearness of the allocation of essentials in the GLCM to the GLCM slanting. It is 1 for a slanting GLCM. A standardized image will effect in a co-occurrence matrix with a mixture of elevated and short P [i, j]'s. A varied image will effect in an even extend of $\mathrm{P}[\mathrm{i}, \mathrm{j}]$ 's. 
13) Distinction:

Distinction $=\sum_{i-0}^{G-1 G-1} \sum_{j-0}^{G-1}|i-j| \times p(i, j)$

It is determined to find constancy between two groups.

14) Diversity in variance:

$$
\operatorname{var}=\sum_{i-0}^{G-1 G-1} \sum_{j-0}^{G-1}(i-\mu)^{2} \times p(i, j)
$$

It is the summation of divergence among greatness of the middle pixel and its locality. The GLCM feature is tabulated in Figure 6.

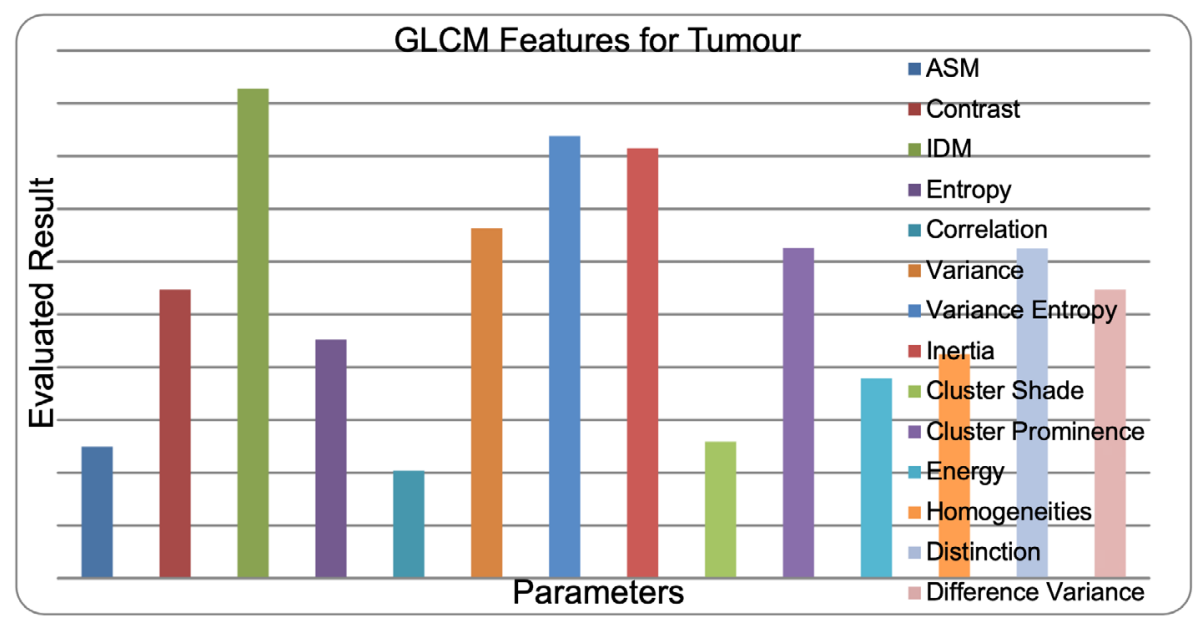

Figure 6. GLCM Features performance for Tumour.

Suorce: own elaboration.

Local Binary Patterns: the LBP feature vector is fashioned in the following manner:

- The inspected space is segregated into chambers (e.g. 16x16 pixels for each chamber).

- For every pixel in a chamber, evaluate the pixel to each one of its 8 neighbors (on its left-top, left-middle, left-bottom, right-top, etc.) either in clockwise or counterclockwise. 
- If the middle pixel's value is superior to the nearby value then replace with " 1 ". If not, replace with " 0 ". Thus 8-digit binary number (which is generally changed to decimal).

- Histogram is computed over the cell (i.e., arrangement of pixels are lesser or larger than the midpoint) and feature vector of the box is obtained.

- The feature vector can be trained using the Support Vector Machine (SVM) and ANFIS to classify images.

\subsubsection{TUMOR DETECTION USING SVM CLASSIFIER}

SVM fundamentally tries to split the given data into decision plane. Decision plane is a hyper plane which splits the data into two classes. Training points obtained by the feature vectors are the supporting vector which describes the hyper plane. The training data $\mathrm{x}$ contains of $\mathrm{n}$ data trials each of $\mathrm{m}$ dimensions and fitting to class $\mathrm{y}$, is expressed as:

$$
\left(x_{1}, y_{1}\right) \ldots\left(x_{i}, y_{i}\right) \ldots\left(x_{n}, y_{n}\right), x \xi R^{m}, y \xi\{+1,-1\}
$$

Table 1. GLCM Matrixfor Tumor Results.

\begin{tabular}{|c|c|}
\hline PARAMETERS & RESULTS \\
\hline ASM & 2.49 \\
\hline Contrast & 5.47 \\
\hline IDM & 9.28 \\
\hline Entropy & 4.52 \\
\hline Correlation & 2.04 \\
\hline Variance & 6.63 \\
\hline Variance Entropy & 8.38 \\
\hline Inertia & 8.15 \\
\hline Cluster Shade & 2.59 \\
\hline
\end{tabular}




\begin{tabular}{|c|c|}
\hline $\begin{array}{c}\text { Cluster } \\
\text { Prominence }\end{array}$ & 6.26 \\
\hline Energy & 3.79 \\
\hline Homogeneities & 4.25 \\
\hline Distinction & 6.25 \\
\hline $\begin{array}{c}\text { Difference } \\
\text { Variance }\end{array}$ & 5.47 \\
\hline
\end{tabular}

Source: own elaboration.

SVM assigns data $(\mathrm{xi}, \mathrm{y})$ into an infinitedimensional hyper plane $(\mathrm{xi}, \mathrm{y})$ by using Gaussian kernel function and describes its rule decision as sign $(\mathrm{f}(\mathrm{x}))$. The discriminant function $\mathrm{f}(\mathrm{x})$ produces the optimal hyper plane decision border by using weight vector $\mathrm{w}^{*}$ and bias $\mathrm{b}^{*}$.

$$
f(x)=w^{*} \phi(x)+b^{*}
$$

The optimal values of $\mathrm{w}^{*}$ and $\mathrm{b}$ are assessed by explaining the following optimization problem.

$$
\left.\min _{w, \xi}\left\{\frac{1}{2} w^{2}+C \sum_{i=1}^{n} \xi_{i}\right\} ; y_{i}\left(w . \phi\left(x_{i}\right)+b\right)\right) \geq 1-\xi_{i}, \xi_{i} \geq 0
$$

where, $\mathrm{C}$ is regularization constraint, which lastly produces optimum level for weight vector $\mathrm{w}^{*}$ and bias $\mathrm{b}^{*}$. Figure 7 represents the malignant brain tumour identified image by using SVM classifier.

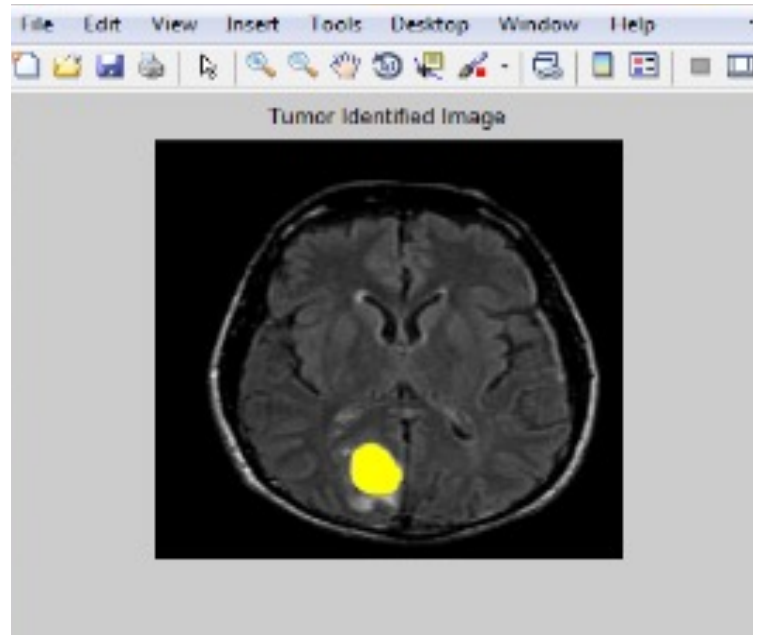




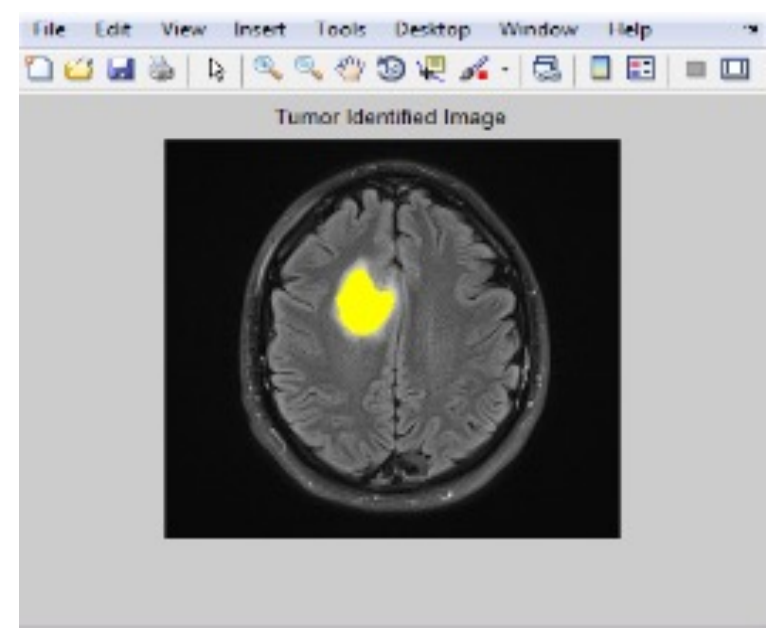

Figure 7. Tumour Identified Image.

Suorce: own elaboration.

\subsection{PROPOSED FOR IDENTIFYING STROKE}

If the tested MRI image intensity level is below 200, it will detect stroke otherwise to detect tumour. The following steps will be carried in stroke segmentation as shown in Figure 4. All process are same except the classifier.

- Noise and skull removal in pre processing.

- K-means segmentation.

- Feature Extraction using GLGM.

- Stroke detection using ANFIS classifier.

\subsubsection{PRE-PROCESSING-NOISE AND SKULL REMOVAL}

The same preprocessing steps of brain tumor MRI is carried over here The noisy image removed from noise and skull is shown in Figure 8. 


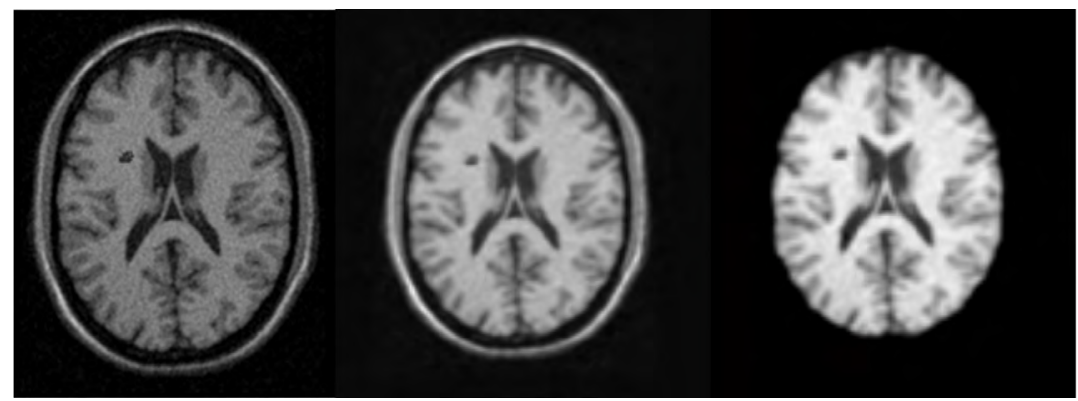

Figure 8. (a) Noise affected image. (b) noise removed image. (c) Skull removed image.

Suorce: own elaboration.

\subsubsection{K-MEANS SEGMENTATION}

$\mathrm{K}$-means clustering targets to divide $\mathrm{n}$ interpretations into $\mathrm{k}$ clusters in which each reflection belongs to the cluster with the near mean, helping as a model of the cluster.

The subsequent steps of K-means:The total number of clusters $\mathrm{k}$ with primary cluster centroid was chosennvi $=1,2, \ldots \mathrm{k}$

Separation of the input statistics points into k clusters with allocating everystatistics element $\mathrm{xj}$ to the neighbouring cluster centroid vi by nominated distance extent. Euclidean distance equation was given as,

$$
d_{i j}=\left\|X_{j}-v_{i}\right\|
$$

Where $\mathrm{X}=\{\mathrm{x} 1, \mathrm{x} 2, \ldots \mathrm{xn}\}$ is the input data set.

Cluster transfer matrix Ui calculated which is the separation of the data points with the binary bias value of the jth data point to the ith cluster such that $U=|u i, j|$ and $u i, j$ in $\{0,1\}$ for all $\mathrm{i}, \mathrm{j}$

$$
\sum_{i-1}^{k} u_{i j}=1 \text { foralljand } 0<\sum_{j-1}^{n} u_{i j}<n \text { for all } 1
$$

Centroid is rearranged using the membership values by 


$$
v_{i}=\frac{\sum_{j-1}^{n} u_{i j} X_{j}}{\sum_{i j-1}^{n} u_{i j}} \text { for all } i
$$

The iteration will be carried over by step 2, until there is no change in the centroid of the cluster from the previous iteration. The k-means clustering process improves the sum-ofsquared-error-based objective function $\mathrm{Jw}(\mathrm{u}, \mathrm{v})$ then

$$
J_{w}(u, v)=\sum_{i=1}^{n} \sum_{j-1}^{n}\left\|X_{j}-v_{i}\right\|^{2}
$$

The projected method K-means clustering is exact hopeful algorithm for clustering formation from Ischemic stroke MR image. To test our projected technique a Magnetic Resonance Imaging (MRI) image of human brain was taken. The k-mean segmented image is given in the Figure 9.

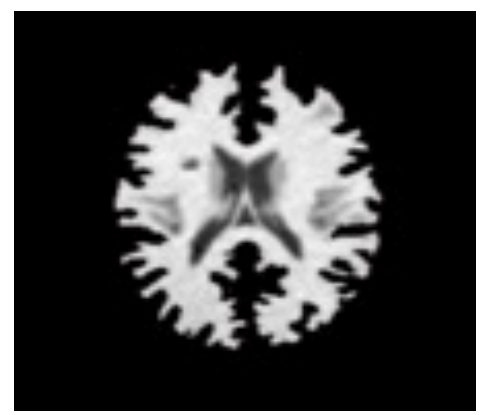

Figure 9. k-means clustering image.

Suorce: own elaboration.

\subsubsection{FEATURE EXTRACTION USING GLCM}

The feature extraction is carried as in brain tumour MRI. The features are tabulated in Table 2. In Figure 10 GLCM features analysis of stroke graph is provided. 


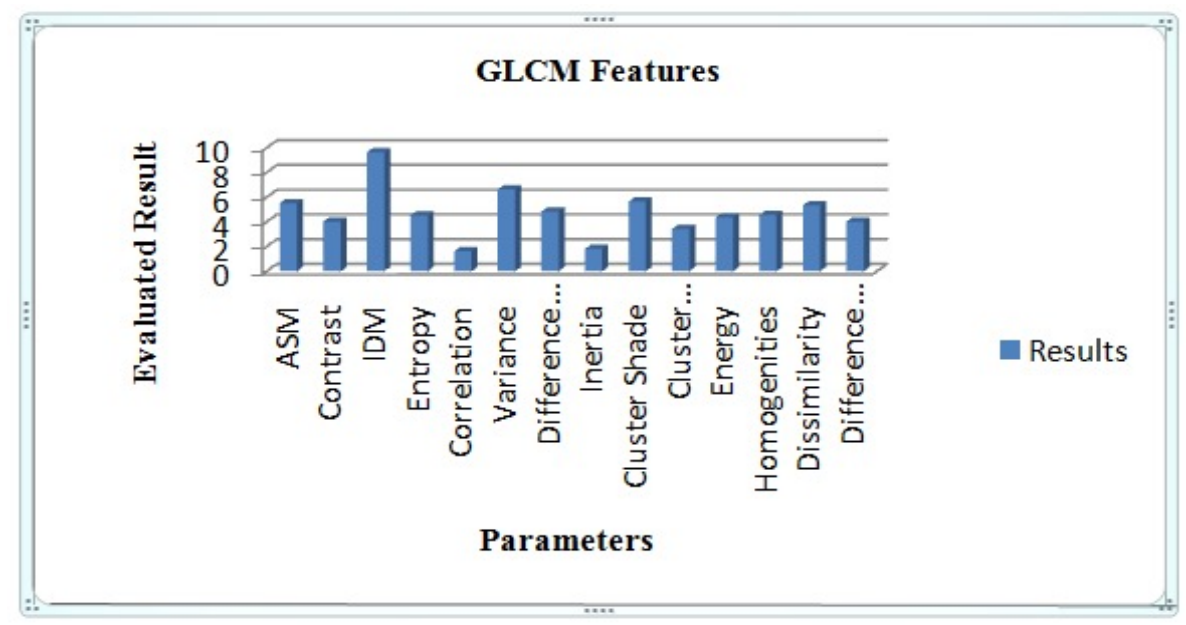

Figure 10. GLCM Features performance for stroke.

Suorce: own elaboration

Table 2. GLCM Matrix for Stroke Results.

\begin{tabular}{|c|c|}
\hline PARAMETERS & RESULTS \\
\hline ASM & 5.51 \\
\hline Contrast & 4.02 \\
\hline IDM & 9.63 \\
\hline Entropy & 4.52 \\
\hline Correlation & 1.62 \\
\hline Variance & 6.63 \\
\hline Variance Entropy & 4.83 \\
\hline Inertia & 1.81 \\
\hline Cluster Shade & 5.64 \\
\hline $\begin{array}{c}\text { Cluster } \\
\text { Prominence }\end{array}$ & 3.43 \\
\hline Energy & 4.35 \\
\hline Homogeneities & 4.55 \\
\hline Distinction & 5.33 \\
\hline $\begin{array}{l}\text { Difference } \\
\text { Variance }\end{array}$ & 4.02 \\
\hline
\end{tabular}

Suorce: own elaboration. 
$\underline{\text { Stroke detection using ANFIS classifier }}$

It uses hybrid knowledge technique that proposes ANFIS to build an input-output plotting model. In the simulation, the ANFIS architecture uses IF THEN rules.

\section{ANFIS Architecture}

Assume - two inputs $\mathrm{X}$ and $\mathrm{Y}$ and one output $\mathrm{Z}$

Rule 1: If $\mathrm{x}$ is $\mathrm{Al}$ and $\mathrm{y}$ is $\mathrm{B} 1$,

then $\mathrm{fl}=\mathrm{pl} \mathrm{x}+\mathrm{q} l \mathrm{y}+\mathrm{rl}$

Rule 2:If $\mathrm{x}$ is $\mathrm{A} 2$ and $\mathrm{y}$ is $\mathrm{B} 2$,

then $\mathrm{f} 2=\mathrm{p} 2 \mathrm{x}+\mathrm{q} 2 \mathrm{y}+\mathrm{r} 2$

$\underline{\text { Layer } 1}$

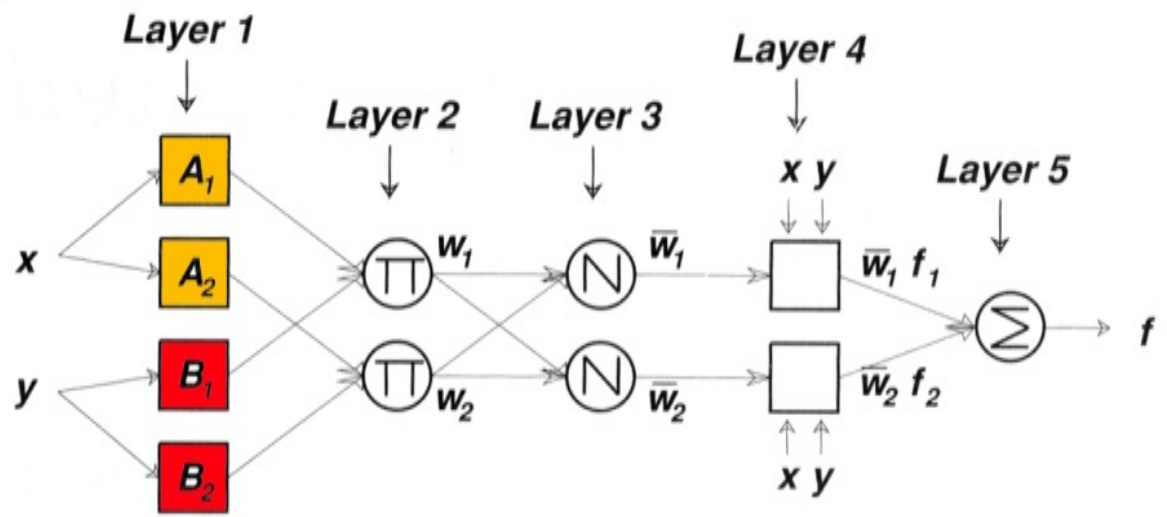

Figure 11. Layer Architecture of ANFIS.

Suorce: own elaboration.

Every node $\mathrm{i}$ in this layer is an adaptive node with a node function, $\mathrm{O} 1, \mathrm{i}=\mathrm{mAi}(\mathrm{x})$, for $\mathrm{I}=$ 1,2 , or $\mathrm{O} 1, \mathrm{i}=\mathrm{mBi}-2(\mathrm{y})$, for $\mathrm{I}=3,4$ 


\section{Layer 2}

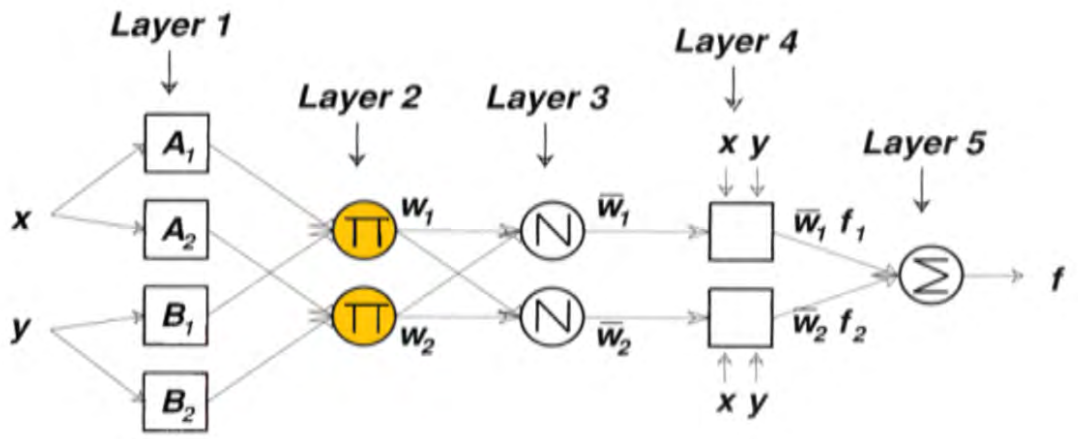

Figure 12. 2nd layer Architecture of ANFIS.

Suorce: own elaboration.

Every node $\mathrm{i}$ in 2nd layer is a fixed node labeled $\prod$, whose yield is the multiplication of all the incoming signals:

$\mathrm{O} 2, \mathrm{i}=\mathrm{Wi}=\min \{\mathrm{mAi}(\mathrm{x}), \mathrm{mBi}(\mathrm{y})\}, \mathrm{i}=1,2$

Each node output characterizes the firing asset of an instruction.

\section{$\underline{\text { Layer } 3}$}

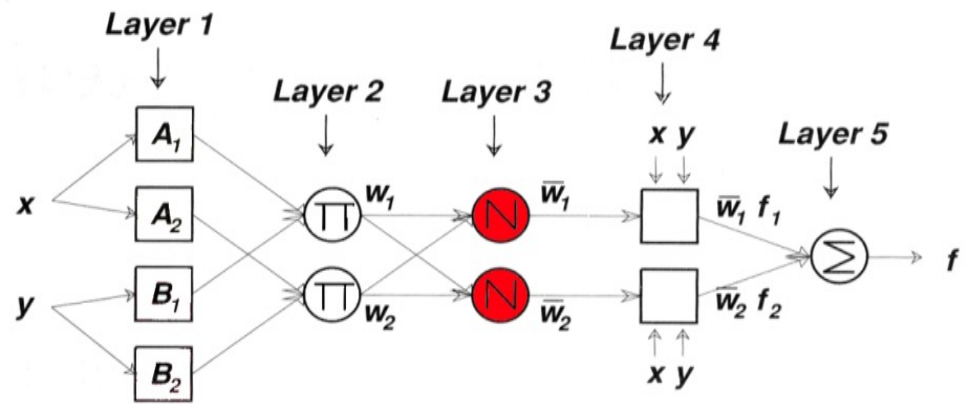

Figure 13. 3rd layer Architecture of ANFIS.

Suorce: own elaboration.

Every node in 3rd layer is a fixed node labeled $\mathrm{N}$.

$\mathrm{O} 3, \mathrm{i}=\mathrm{Wi}=\mathrm{Wi} /(\mathrm{W} 1+\mathrm{W} 2), \mathrm{i}=1,2$ 
The ith node estimates the proportion of the ith instruction's firing asset to the sum of all rules'firingstregths.

\section{Layer 4}

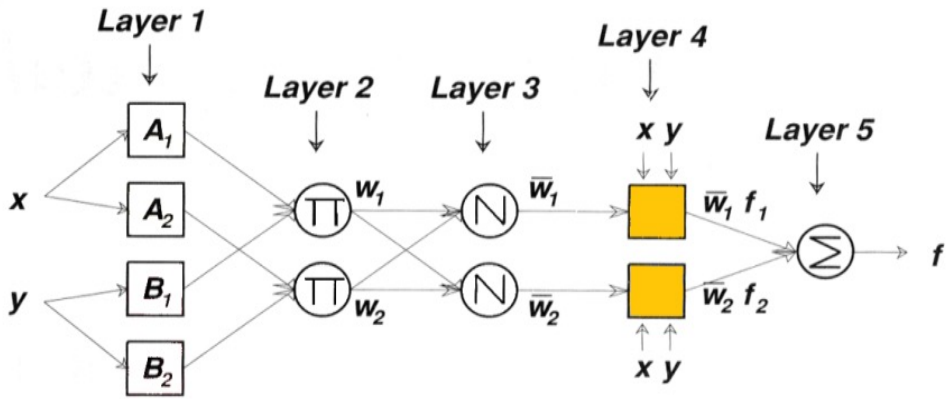

Figure 14-15. 4th layer Architecture of ANFIS.

Suorce: own elaboration.

Every node $\mathrm{i}$ in this layer is an adaptive node with a node function,

$\mathrm{O} 4, \mathrm{i}=\overline{\text { wi }}$ fi $=\overline{\text { wi }}(\mathrm{pix}+$ qiy + ri $) \ldots$ Consequent parameters.

\section{$\underline{\text { Layer } 5}$}

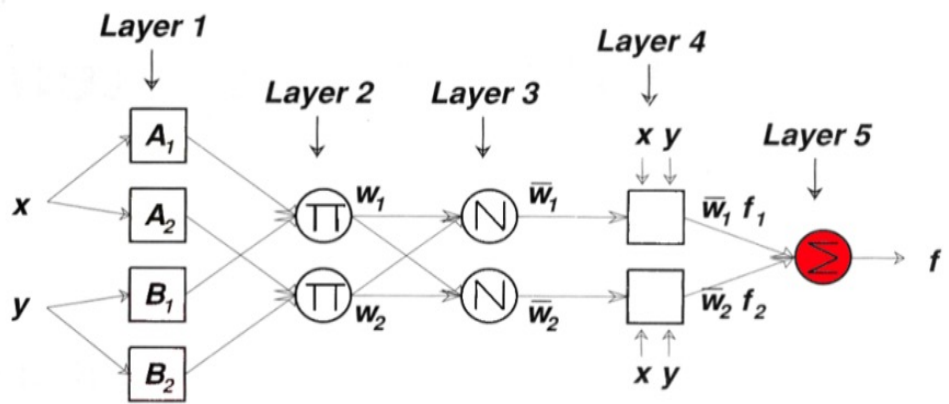

Figure 15. 5th layer Architecture of ANFIS.

Suorce: own elaboration.

The nodes in this layer is a stable node labeled $\mathrm{S}$, used to calculate the complete yield as the summation of all received signals.

O5, $1=$ Siwifi.

By using ANFIS classifier, stroke can be detected and is shown in Figure 16. 


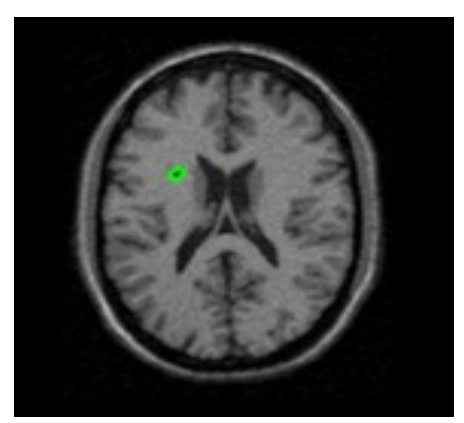

Figure 16. Stroke Identified Image.

Suorce: own elaboration.

\section{RESULTS}

\subsection{PERFORMANCE ANALYSIS FOR STROKE USING ANFIS CLASSIFIER}

The classifier namely ANFIS had established to ischemic brain and normal brain. The performance of the classifier in provisions of sensitivity, specificity and accuracy was estimated. The performances are tabulated in Table 3. The accuracy obtained in 0.99 using ANFIS classifier.

Human brain stroke can be diagnose using this proposed system. Accuracy of 0.998428 isobtained by ANFIS classifier. In Figure 17 performance analysis graph is given using ANFIS classifier.

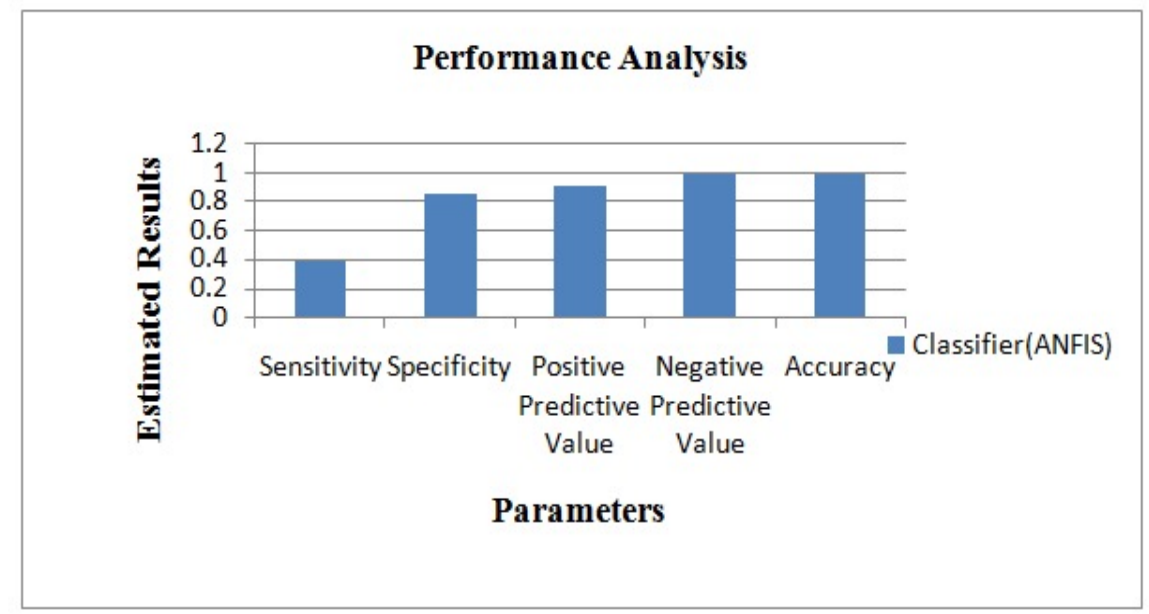

Figure 17. Performance Evaluation Graph in Stroke.

Suorce: own elaboration 


\subsection{PERFORMANCE ANALYSIS OF TUMOUR}

The SVM Classifiers had established to classify benign brain tumour and malignant brain tumour. The performance of the classifiers in provisions of similarity index, overlap fraction and Extra fraction was estimated.

Three quantitative events to calculate in the region of the segmentation result are Similarity Index (SI), Overlap fraction (OF), and Extra Fraction (EF).

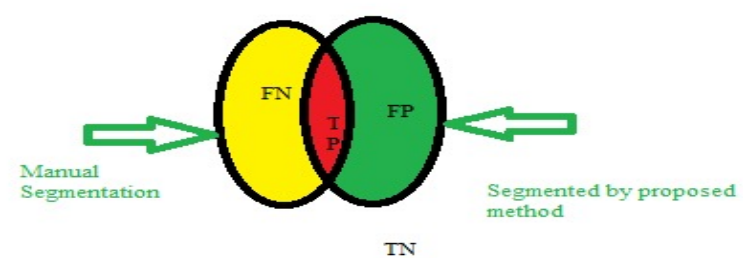

Figure 18. Comparison between the various performance parameter by proposed segmented regions and manual segmentation.

Suorce: own elaboration.

Figure 18 shows that TP is the amount of true-positive pixels identified by manual segmentation and proposed method, FP is the amount of false-positive pixels noticed by manual segmentation and proposed method, and FN is the amount of false-negative pixels comparative to the tumourarea with manual segmentation but it is not identified by the proposed method.

Overlap Fraction $=\frac{T P}{T P+F N}$

$=\frac{F P}{T P+F N}$

Similarity Index $=\frac{2 \times T P}{2 \times T P+F P+F N}$

In a worthy segmentation Similarity Index (SI), Overlap Fraction $(\mathrm{OF})$ which are achieved by equations (36), (34) should be high and Extra fraction (EF) obtained by equation (35) 
should be low. The performances are tabulated in Table 3. In Figure 17 performance analysis graphs are given.

Table 3. Segmentation.

\begin{tabular}{|c|c|c|c|}
\hline PARAMETERS & $\begin{array}{c}\text { SIMILARITY INDEX } \\
\text { (SI) }\end{array}$ & $\begin{array}{c}\text { OVERLAP FRACTION } \\
\text { (OF) }\end{array}$ & $\begin{array}{c}\text { EXTRA FRACTION } \\
\text { (EF) }\end{array}$ \\
\hline Tumour Image set 1 & 0.785531 & 0.783523 & 0.211364 \\
\hline Tumour Image set 2 & 0.766866 & 0.766866 & 0.233134 \\
\hline Average & 0.776198 & 0.775194 & 0.222249 \\
\hline
\end{tabular}

Suorce: own elaboration.

\section{DISCUSSION}

Our projected method is executed in MATLAB. The projected method has been tried on dataset of real brain MR images containing of normal and tumor brain images. Hybrid classifier for detecting abnormalities in brain has been proposed. The abnormality may be stroke or brain tumour. Separate algorithm will be used for detecting brain tumour and stroke. In the projected method, the abnormality region is segmented and it is treated as test image. Based on the intensity level of the abnormalities, either it will detect stroke or tumour. If the threshold value is less than 200 then it will be considered as stroke brain MRI. Then pre-processing and skull removal of image is carried out. After which the segmentation is passed out by using K-means algorithm. Then from the segmented result, features are extracted using GLCM matrix in order to train the ANFIS classifier and performance analysis is carried out. If the threshold value is above 200 then it will be considered as tumour brain MRI. Then pre-processing and edge detection of image is carried out by using anisotropic filter and canny edge detections method. After which the segmentation is passed out by K-means algorithm. Features are extracted using GLCM matrix and LBP in order to train the SVM classifier and performance analysis is carried out in terms of Similarity Index (SI), Overlap Fraction (OF), and Extra Fraction (EF). 


\section{CONCLUSIONS}

The proposal of image segmentation and classification appears to be an interesting task, as the medical images are exposed to noise. In particular anisotropic filter based image denoising scheme proves to be an efficient method for noise removal. Computer aided detection system helps in detecting ischemic stroke and malignant brain tumor has been developed. This system detects to generate accurate quantitative results. The proposed system helps the physicians to diagnose human brain stroke. Accuracy of 0.999 , sensitivity 0.38 , specificity 0.86, PPV 0.91, NPV 0.99 is obtained by ANFIS classifier. Three quantitative events to calculate brain tumor of average segmentation results: Similarity Index (SI), Overlap fraction $(\mathrm{OF})$, and Extra Fraction $(\mathrm{EF})$ is $0.776194,0.775198,0.222213$ is obtained by SVM classifier. It usually aided to progress the accuracy in detection of malignant brain tumor.

\section{ACKNOWLEDGEMENT}

We would like to thank our institution Kalasalingam Academy of Research and Education for support.

\section{REFERENCES}

Adams, H., Adams, R., Zoppo, G. del, \& Goldstein, L. B. (2005). Guidelines for the early management of patients with ischemicstroke, guidelines update, a scientific statement from the Stroke Council of the American Heart Association/ American Stroke Association. Stroke, 36(2), 916-921. https://doi.org/10.1161/01. STR.0000163257.66207.2d

Amutha, C., \& Rajagopalan, S. P. (2013). Brain stroke classification based on MultiLayer Perceptron using Watershed segmentation. Journal of Theoretical and Applied Information Technology, 56(2), 410-416. https://www.researchgate.net/publication/285940599_ Brain_stroke_classification_based_on_multi-layer_perceptron_using_watershed_ segmentation_and_gabor_filter 
Bala, A. (2012). An Improved Watershed Image Segmentation Technique using MATLAB. International Journal of Scientific E Engineering Research, 3(6), 5-8. https://www.ijser. $\mathrm{org} /$ researchpaper/an-improved-watershed-image-segmentation-technique-usingmatlab.pdf

Bian, W., Hess, G. P., Chang, S. M., Nelson, S. J., \& Lupo, J. M. (2013). Computeraided detection of radiation-induced cerebral micro bleeds on susceptibilityweighted MR images. NeuroImage: Clinical, 2, 282-290. https://pubmed.ncbi.nlm. nih.gov/24179783/

Dzialowski, I., Hill, M. D., Coutts, S. B., Demchuk, A. M., Kent, D. M., Wunderlich, O., \& von Kummer, R. (2012). Extent of Early Ischemic Changes on Computed Tomography (CT) Before Thrombolysis Prognostic, Value of the Alberta Stroke Program Early CT Score in ECASS II. Stroke, 37(4),973-978. https:// doi.org/10.1161/01.STR.0000206215.62441.56

Ford, A. L., An, H., Vo, K. D., Lin, W., \& Lee, J.-M. (2012). Defining the Ischemic penumbra using hyper active neuroimaging: driving quantitative Ischemic Thresholds. Translational Stroke Research, 3(2), 198-204. https://pubmed.ncbi.nlm.nih. gov/24323775/

Grady, L. (2006). Random Walks for Image Segmentation. IEEE Transactionson Pattern Analysis and Machine Intelligence, 28(11), 1768-1783. https://ieeexplore.ieee.org/ document/1704833

Grady, L., \& Schwartz, E. L. (2006). Isoperimetric Graph Partitioning for Image Segmentation. IEEE Transactionson Pattern Analysis and Machine Intelligence, 28(3), 469475. https://ieeexplore.ieee.org/document/1580491

Hema, N., \& Bhavani, R. (2013). Computer aided detection of ischemic stroke using segmentation and texture features. Measurement, (46), 1865-1874. https:/ / www.infona. pl/resource/bwmeta1.element.elsevier-6cea82e0-3e95-37c1-81e7-b48eb7905e72

Kharat, K. D., \& Kulkarni, P. P. (2012). Brain Tumor Classification Using Neural Network Based Methods. International Fournal of Computer Science and Informatics 
ISSN (PRINT), 1(4), 2231-5292. https://www.interscience.in/cgi/viewcontent. cgi? article $=1075 \&$ context $=$ ijcsi

Mahmoud-Ghoneim, D., Toussaint, G., Constans, J.-M., \& Certaines, J. D. de. (2003). Three dimensional texture analysis in MRI: a preliminary evaluation in gliomas. Magnetic Resonance Imaging, 21(9), 983-987. https:/ / doi.org/10.1016/S0730725X(03)00201-7

Rekik, I., Allassonnière, S., Carpenter, T. K., \& Wardlaw, J. M. (2012). Medical image analysis methods in MR/CT-imaged acute-subacute ischemic stroke lesion: Segmentation, prediction and insights into dynamic evolution simulation models. NeuroImage: Clinical, 1(1), 164-178. https://doi.org/10.1016/j.nicl.2012.10.003

Runchey, S., \& McGee, S. (2010). Does This Patient Have a Hemorrhagic Stroke?. JAMA: the journal of the American Medical Association, 303(22), 2280-2286. https:/ / doi. org/10.1001/jama.2010.754

Shi, J., \& Malik, J. (2000). Normalized Cuts and Image Segmentation. IEEE Transactionsonpatternanalysis and machine intelligence, 22(8), 888-905. https://ieeexplore. ieee.org/document/868688

Thom, T., Haase, N., Rosamond, W., Howard, V. J., Rumsfeld, J., Manolio, T., Zheng, Z.-J., Flegal, K., O’Donnell, C., Kittner, S., Lloyd-Jones, D., Goff, D. D., Hong, J., Adams, R., Friday, G., Furie, K., Gorelick, P., Kissela, B., Marler, J., ... \& Wolf, P. (2006). Heartdisease and stroke statistics update: a report from the American Heart Association Statistics Committee and Stroke Statistics Subcommittee. Girculation, 113(6), 85-151. https://pubmed.ncbi.nlm.nih. gov/16407573/

Toni, D., Iweins, F., von Kummer, R., Busse, O., Bogousslavsky, J., Falcou, A. E., Lesaffre, E., \& Lenzi, G. L. (2006). Identification of lacunar in farcts before thrombolysis in the ECASS 1 study. Neurology, 54(2),684-688. https://pubmed.ncbi. nlm.nih.gov/10680804/ 
Udupa, J., \& Samarasekera, S. (1996). Fuzzy Connectedness and Object Definition: Theory, Algorithms, and Applications in Image Segmentation. CVGIP Graph. Model. Image Process., 58, 246-261. https://www.semanticscholar.org/paper/FuzzyConnectedness-and-Object-Definition \%3A-Theory\%2C-Udupa-Samarasekera/4a 3e9fcfc88d53cdf0b7633fbc914f98dle92225

Von Kummer, R. (2005). The impact of CT on acute stroke treatment. In P. Lyden (ed.), Thrombolytic Therapy for Stroke. Humana Press, pp. 249-278. 